\title{
Space-time optical imaging framework: Holographic particle tracking velocimetry
}

\author{
Ni Chen, Congli Wang, and Wolfgang Heidrich \\ Visual Computing Center, King Abdullah University of Science and Technology, Thuwal 23955, Saudi Arabia \\ ni.chen@kaust.edu.sa
}

\begin{abstract}
We present a joint optimization framework for four-dimensional (4D) optical imaging: volumes and movements are reconstructed jointly in a higher space-time dimension, enabling faster convergence and better reconstruction quality of both the volumes and movements within a few minutes on modern GPUs. We verified the framework on digital holographic particle tracking velocimetry, a typical 4D optical imaging example. Experimental results are presented to show the efficiency of the proposed technique. (C) 2021 The Author(s)
\end{abstract}

\section{Theory}

The temporal evolution of a volume $o$ defines an apparent movement of the volume, known as fluid velocities. Motion flows can be numerically reconstructed from consecutive, rapid measurements of changing particle volumes. Under the assumption of incompressibility, i.e., divergence-free flow, the motion flow $\mathbf{v}_{t} \in \mathbb{R}^{3}$ relates temporally neighboring particle volumes from time $t$ to $t+1$ [1]. That is, with $\mathbf{x}=(\mathbf{r}, z)$ :

$$
o_{t}\left(\mathbf{x}+\mathbf{v}_{t}\right) \approx o_{t+1}(\mathbf{x}),
$$

where subscripts $(t$ and $t+1)$ denote for two neighboring time frames. Given a sequence of indexed 2D images $b_{t}(\mathbf{r})$ of $t=1,2, \cdots, T$, at each frame $t$, we would like to recover simultaneously its volume $o_{t}(\mathbf{x})$ and the associated $3 \mathrm{D}$ flow vector $\mathbf{v}_{t}(\mathbf{x})$. By taking into consideration of the temporal coherence between neighboring frames, we jointly estimate volumes and fluid flows and this strategy optimizes for neighboring frames together, and hence closing the numerical reconstruction loop. These yield the following optimization problem:

$$
\min _{\mathbf{o}, \mathbf{v}} \sum_{t=1}^{T}\left\|\mathbf{A} \mathbf{o}_{t}-\mathbf{b}_{t}\right\|_{2}^{2}+\tau \sum_{t=1}^{T-1}\left\|\mathbf{o}_{t+1}(\mathbf{x})-\mathbf{o}_{t}\left(\mathbf{x}+\mathbf{v}_{t}\right)\right\|_{2}^{2}+\operatorname{priors}(\mathbf{o})+\operatorname{priors}(\mathbf{v}),
$$

where priors $(\mathbf{v})$ may represent the smoothness and incompressible property [1,2] of the flow, and priors $(\mathbf{o})$ represents the property of the volumes, usually is sparsity. Specifically, Eq. (2) is solved by alternating between solving particle volume $\mathbf{o}$ and solving volumetric movement flow $\mathbf{v}$, as in Algorithm 1:

$$
\begin{array}{ll}
\min _{\mathbf{o}} & \sum_{t=1}^{T}\left\|\mathbf{A} \mathbf{o}_{t}-\mathbf{b}_{t}\right\|_{2}^{2}+\tau \sum_{t=1}^{T-1}\left\|\mathbf{o}_{t+1}(\mathbf{x})-\mathbf{o}_{t}\left(\mathbf{x}+\mathbf{v}_{t}\right)\right\|_{2}^{2}+\operatorname{priors}(\mathbf{o}), \\
\min _{\mathbf{v}} & \tau \sum_{t=1}^{T-1}\left\|\mathbf{o}_{t+1}(\mathbf{x})-\mathbf{o}_{t}\left(\mathbf{x}+\mathbf{v}_{t}\right)\right\|_{2}^{2}+\operatorname{priors}(\mathbf{v}) .
\end{array}
$$

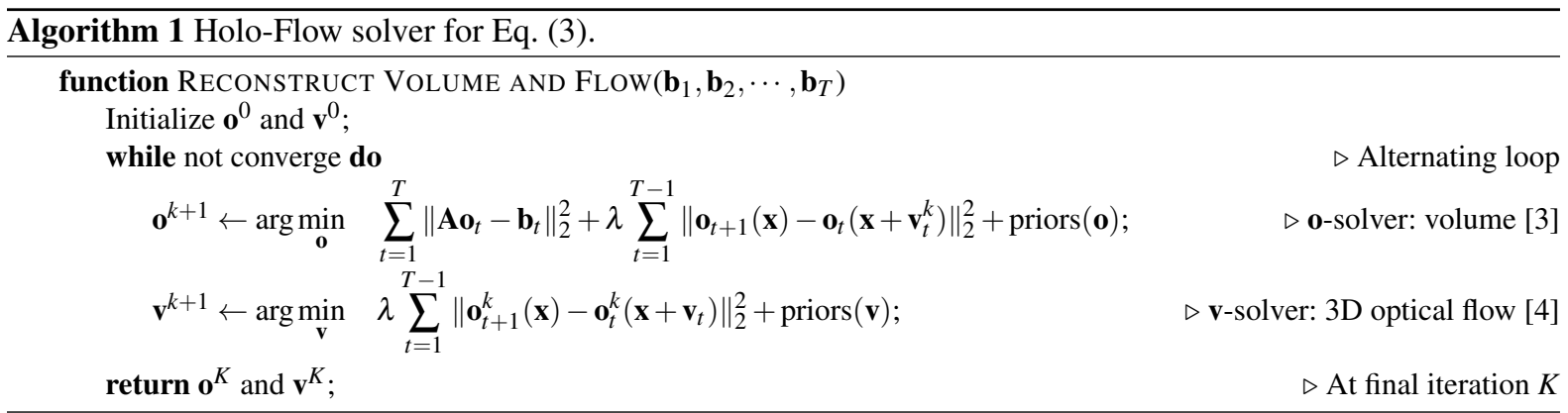

In Eq. (2) and Eq. (3), A can be an optical system of holography or light field microscopy, where $b_{t}(\mathbf{r})$ are holograms or light field images. We take the holographic particle tracking velocimetry (HPTV) as an example. 
Specifically, we solve particles using FASTA iterative shrinkage algorithm [3], and solve flow using standard HornSchunck optical flow [4]. In the following, we evaluate our proposed approach on HPTV based on experimental hologram data.

\section{Verification of the proposed technique with holographic PTV}

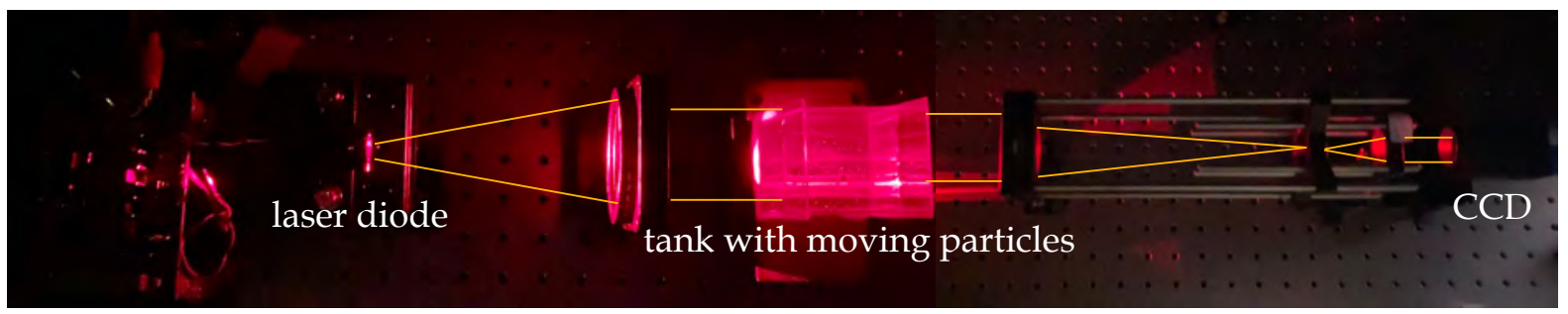

(a) Experimental setup.

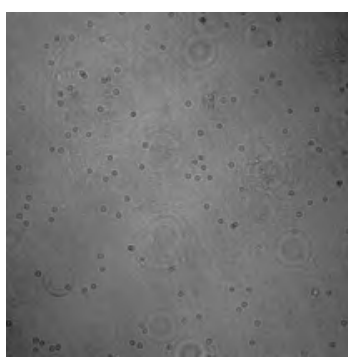

(b) Selected hologram frames.

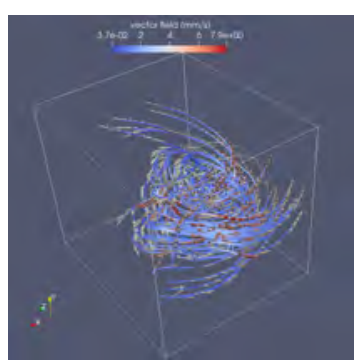

(c) Reconstructed fluid flow.

Fig. 1: Verification of the proposed technique with holographic PTV.

In the experiment, a tank containing seeded particles in high viscosity liquid was illuminated by a laser beam, as shown in Figure 1(a). We spun the water anticlockwisely from top of the tank and captured hologram sequences through a 4F system that consists of two achromatic lenses (focal length of $200 \mathrm{~mm}$ and $60 \mathrm{~mm}$ ), as shown in Figure 1(a). In the flow reconstruction, we downsampled the holograms by a factor of 8 from an original resolution of $2048 \times 2048$ to $256 \times 256$. The depth was discretized into 101 layers $(0.1 \mathrm{~mm} / \mathrm{layer}$ with a voxel resolution of $55.2 \mu \mathrm{m} \times 55.2 \mu \mathrm{m} \times 100 \mu \mathrm{m}$. The path-line visualization of the recovered flow field is shown in Figure 1(c). As expected, the swirl rotates clock-wisely along the $y$ axis. The reconstruction only shows a part of the swirl because of the limited field of view of the experiment (around $23.6 \mathrm{~mm}$ ), about one-half of the total tank width. The recovered flow field shown in Figure 1 is consistent with our expectation and the observed hologram frames. For the experiment, the outer iterations are 3 , and the inner iteration of the hologram solver is 20 , and $\tau=0.001$. Specific prior weights are tuned to plausible values. The reconstruction of the last experiment takes around $1 \mathrm{~min}$ on a Ubuntu 18.04 Linux workstation with 2.70GHz Intel(R) Xeon(R) CPU E5-2680, 62.9GB RAM, and a NVIDIA TITAN X (Pascal) GPU. Roughly $68 \%$ of that time was spent on the data transfer between CPU and GPU.

\section{Funding Information}

This work was supported by the KAUST individual baseline funding.

\section{References}

1. J. Gregson, I. Ihrke, N. Thuerey, and W. Heidrich, "From capture to simulation," ACM Transactions on Graph. 33, 1-11 (2014).

2. J. Xiong, R. Idoughi, A. A. Aguirre-Pablo, A. B. Aljedaani, X. Dun, Q. Fu, S. T. Thoroddsen, and W. Heidrich, "Rainbow particle imaging velocimetry for dense 3d fluid velocity imaging," ACM Transactions on Graph. 36, 1-14 (2017).

3. T. Goldstein, C. Studer, and R. Baraniuk, "A field guide to forward-backward splitting with a fasta implementation," arXiv preprint arXiv:1411.3406 (2014).

4. B. K. Horn and B. G. Schunck, "Determining optical flow," in Techniques and Applications of Image Understanding, , vol. 281 (International Society for Optics and Photonics, 1981), pp. 319-331. 